\title{
Transient cortical blindness due to cerebral fat embolism
}

\author{
Baki Doğan¹, Sina Çoşkun²(1), Leyla Niyaz ${ }^{3} \mathbb{D}$ \\ ${ }^{1}$ Department of Neurology, Medicine Faculty of Ondokuz Mayıs University, Samsun, Turkey \\ ${ }^{2}$ Department of Orthopedics and Traumatology, Medicine Faculty of Ondokuz Mayıs University, Samsun, Turkey \\ ${ }^{3}$ Department of Ophthalmology, Medicine Faculty of Ondokuz Mayıs University, Samsun, Turkey
}

Fat embolism syndrome (FES) is a rare disorder most commonly manifested with respiratory insufficiency following long bone fractures or orthopedic surgery. The reported risk of FES following orthopedic trauma ranges between 1 to $30 \% .{ }^{[1]}$ The classical triad of FES includes respiratory insufficiency, neurological impairment, and petechial skin rash. ${ }^{[2]}$ It usually occurs 24 to $72 \mathrm{~h}$ following the initial insult. ${ }^{[1]}$ The clinical entity in which neurological signs and symptoms predominate is defined as cerebral FES (CFES). Neurological complications of CFES include ischemic or hemorrhagic strokes with focal neurological deficits, headache, apraxia, vision loss, seizures, convulsive and non-convulsive status epilepticus, autonomic dysfunction, acute encephalopathy, confusion, and coma. ${ }^{[3,4]}$ Given the wide range of clinical signs and symptoms, the diagnosis depends on excluding other causes in high-risk patients with a history of trauma and surgery.

Received: December 02, 2021

Accepted: January 19, 2022

Published online: February 10, 2022

Correspondence: Baki Doğan, MD. Ondokuz Mayıs Üniversitesi Tıp Fakültesi Nöroloji Anabilim Dalı, 55280 Atakum, Samsun, Türkiye.

E-mail: dr.bakidgn@gmail.com

Doi: $10.52312 /$ jdrscr.2022.43

Citation: Doğan B, Çoşkun S, Niyaz L. Transient cortical blindness due to cerebral fat embolism. Jt Dis Relat Surg Case Rep 2022;1(2):51-54.

C2022 All right reserved by the Turkish Joint Diseases Foundation

This is an open access article under the terms of the Creative Commons Attribution-NonCommercial License, which permits use, distribution and reproduction in any medium, provided the original work is properly cited and is not used for commercial purposes (http://creativecommons.org/licenses/by-nc/4.0/).

https://www.casereportsjointdrs.org

\section{ABSTRACT}

Fat embolism syndrome (FES) is a disease that manifests with respiratory distress, neurological impairment, and petechial rash and develops due to embolization of fat particles into several organs after long bone fractures or orthopedic surgery. A 26-year-old male patient developed sudden vision loss $24 \mathrm{~h}$ after the tibial fracture. Except for visual acuity, other neurological and ophthalmological examinations were normal. He had transient cortical blindness due to central fat embolism secondary to dislocated tibia fracture. Although the major diagnostic criteria of FES include at least two skin, pulmonary and neurological involvements, cerebral fat embolism may occur alone without any additional organ involvement. Multiple brain fat embolism may lead to cortical blindness, which is usually transient.

Keywords: Cortical blindness, fat embolism syndrome, tibial fracture.

In this article, we report a case of CFES with transient cortical blindness secondary to an unstable tibia fracture which was managed successfully with complete recovery.

\section{CASE REPORT}

A 26-year-old male patient was admitted to the emergency room with a tibial fracture after a motorcycle accident without head trauma. On physical examination, his Glasgow Coma Scale score was 15 . He was alert, and his vital signs were normal. The right knee joint movements were minimally limited, and there was swelling in the right calf. The posterior tibial pulse was palpable, and there was no deficit in the neurological examination. Cranial, thoracic, abdominal, and pelvic injuries were ruled out clinically and radiologically. A right tibial shaft fracture was observed on the X-ray (Figure 1). The leg was stabilized with a long splint, and he was hospitalized with a surgery plan. 

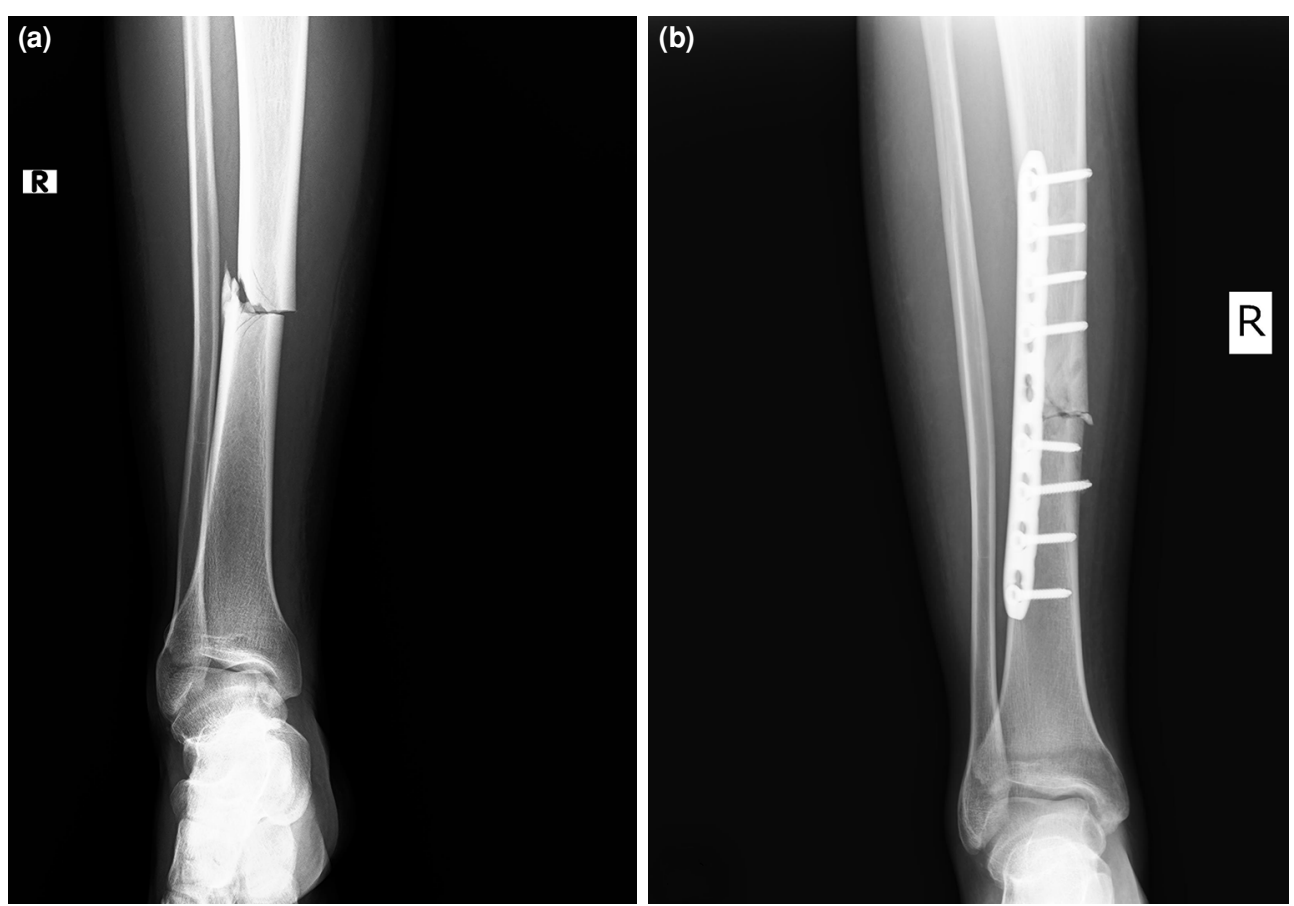

Figure 1. A right tibial shaft fracture was observed on the X-ray (a) preoperative and (b) postoperative.

He complained of sudden vision loss $24 \mathrm{~h}$ after the accident. Ophthalmological examination revealed a severe vision loss at the level of light perception on both eyes. Pupillary light reflex was reactive. Anterior segment and fundus examinations were normal. There were no retinal hemorrhages, detachment, or vascular occlusion. Neurological examination and mental status were utterly normal except for visual loss, which was noticeable totally by the patient. There was no petechial rash or any respiratory symptom or finding of insufficiency. Cranial magnetic resonance imaging (MRI) revealed multiple tiny cortical and subcortical restriction areas of diffusion on both hemispheres suggestive of cerebral embolism interpreted as a "starfield" appearance. Axial diffusion-weighted imaging (DWI) and corresponding apparent diffusion coefficient (ADC) images demonstrate diffusion-restricted lesions in the bilateral cortex and centrum semiovale. Fluid-attenuated inversion recovery (FLAIR) and susceptibility-weighted imaging (SWI) showed no findings suggestive of contusion or diffuse axonal damage. It was evaluated as normal (Figure 2). No epileptiform anomaly or encephalopathy was observed on electroencephalogram. Transthoracic echocardiography was normal with an ejection fraction of $65 \%$, and there was no evidence of patent foramen ovale or thrombus over the valves and in heart cavities. Repeated chest X-ray and electrocardiogram were normal.

The patient was diagnosed with cortical blindness secondary to fat embolism. Anticoagulation treatment with low-molecular-weight heparin was started for cerebral embolism. He was followed closely in terms of mortal complications of fat embolism. As the hemodynamics and vitals were stable after $24 \mathrm{~h}$ of follow-up, the patient underwent orthopedic surgery. The tibial fracture was fixed with a titanium plate. On postoperative Day 2, the visual acuity turned back to normal completely without any visual field deficit. The patient was discharged with anticoagulant therapy after four days of follow-up. On subsequent examination one month later, tibial fracture stabilization was achieved, and there was no neurological or ophthalmological complaint or finding.

The patient was informed that data from the case would be submitted for publication and gave his consent.

\section{DISCUSSION}

Fat embolism syndrome is a lethal disease that occurs mainly after the dislocated long bone fractures. It is also reported after spinal surgery, liposuction, severe pancreatitis, sickle cell disease, burns, 

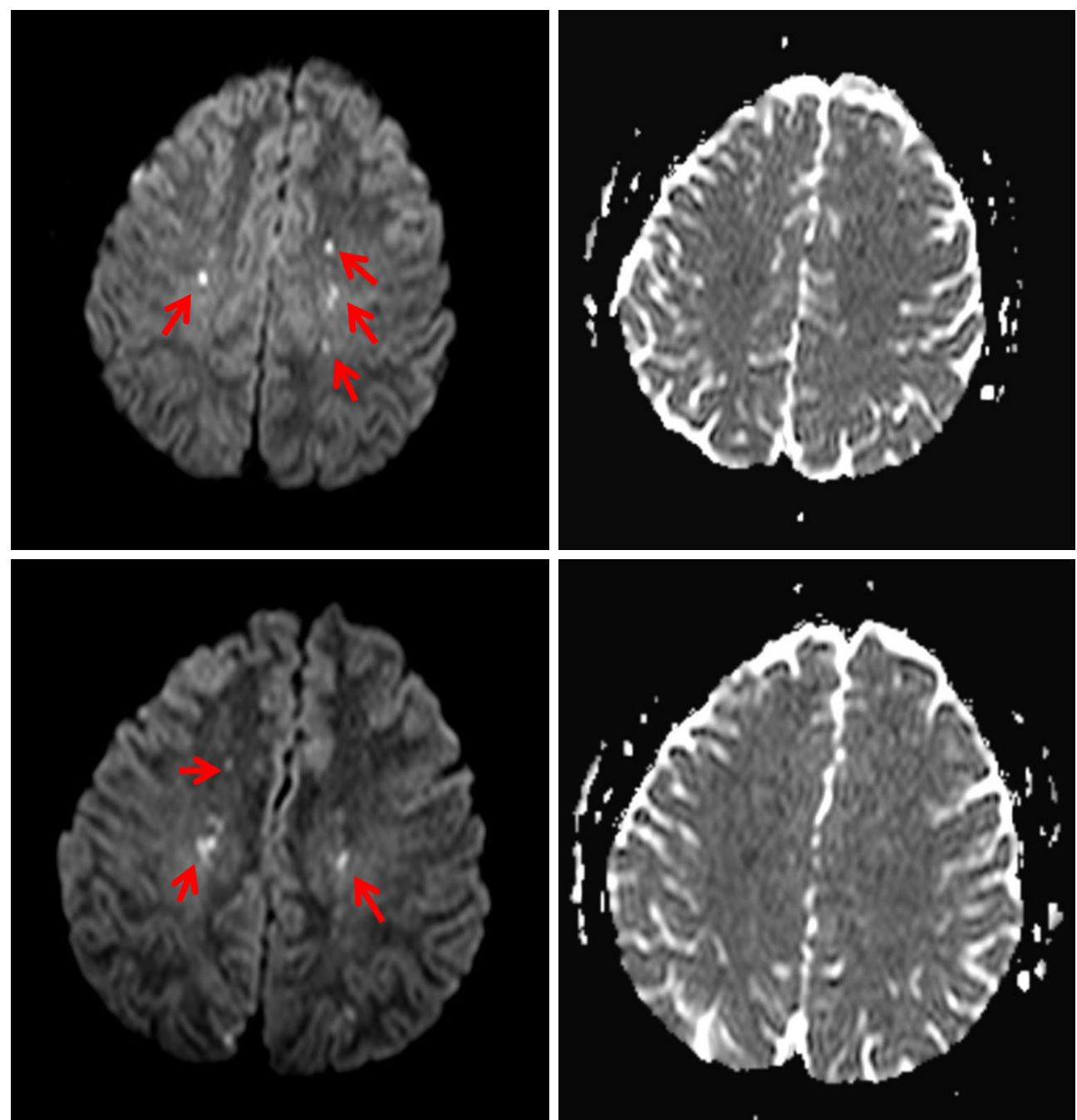

Figure 2. Diffusion weighted MRI brain showing cortical-subcortical DWI hyperintens ADC hypointens lesion in bilateral cerebral hemispheres (Red Arrows show that cerebral embolism interpreted as a "starfield" appearance).

MRI: Magnetic resonance imaging; DWI: Diffusion-weighted imaging; ADC: Apparent diffusion coefficient.

cardiopulmonary bypass surgery, bone marrow harvesting, sternotomy, osteomyelitis, parenteral lipid infusion, tumor lysis syndrome, and fatty liver disease. ${ }^{[5]}$ Systemic FES has a wide range of symptoms and signs. The diagnosis of FES is mainly clinical and is based on specific criteria. Gurd and Wilson ${ }^{[6]}$ proposed definite major and minor criteria for the diagnosis of FES in 1974. Major criteria include skin, respiratory and neurological signs; minor criteria include tachycardia, pyrexia, retinal changes, jaundice, renal dysfunction, increased erythrocyte sedimentation rate, and low hemoglobin, or platelet levels. ${ }^{[6]}$ Currently, for the definite diagnosis of FES, clinicians may choose one from the diagnostic criteria defined by Schonfeld, Gurd, or Lindeque. ${ }^{[1]}$
Our case does not fulfill the classical criteria for the diagnosis of FES. However, sudden visual loss after the bone fracture, normal retinal examination, and bilateral reactive pupils, diffuse multiple cortical and subcortical embolic hyperintensities suggest a CFES. Rapid reversal of symptoms after surgical stabilization of the tibial fracture also leads us to the diagnosis of CFES. Lim et al. ${ }^{[7]}$ reported an unusual case with multiple bone fractures that developed complete bilateral visual loss $2.5 \mathrm{~h}$ after the injury. The ophthalmological examination revealed normal pupillary reflexes with absent retinal pathology. Subsequent MRI showed acute bilateral infarcts in the occipital lobes. The patient's vision improved spontaneously and reached a normal level on 
Day 9 after injury. There were no skin or respiratory manifestations throughout the length of stay in the hospital and, therefore, their patient also did not fulfill the proposed criteria for FES. The diagnosis of FES in such a patient may be based on the close temporal association of the injury and the onset of symptoms together with specific MRI findings and the absence of any other causative accompanying pathology. Another hypothesis for the cerebral lesions may be transient cerebral hypotension caused by the injury at watershed zones. ${ }^{[7]}$

Cortical blindness may be a presentation of cerebral fat embolism. Meena et al. ${ }^{[8]}$ also described a case of cortical blindness $9 \mathrm{~h}$ after the femoral shaft fracture. Subsequently, the patient developed breathlessness and motor aphasia, thus finally satisfying Gurd's criteria. The clinician should pay attention that the typical chronology of symptoms and signs is not always the rule. Neurological symptoms may develop before respiratory symptoms.

Currently, the best medical therapeutic approach for FES is a complete supportive treatment and early fixation of the fracture. Heparin may increase lipid clearance from the intravascular compartment. However, the routine administration of heparin for FES is not recommended..$^{[1]}$ An old double-blinded, randomized trial showed that treatment with methylprednisolone stabilized or reduced serum levels of free fatty acids, maintained arterial oxygen levels, and significantly decreased the risk of FES. The most crucial step for supportive treatment is the early resuscitation and stabilization of hypovolemia to minimize stress. ${ }^{[9]}$

Schemistch et al. ${ }^{[10]}$ found that external fixation or fixation with a plate was shown to produces minor lung injury than intramedullary fixation. Although there is no clear evidence on the relationship between the reamed intramedullary nailing treatment of tibial fractures and the fat embolism, the patient underwent fixation surgery. Surgery was performed open reduction and plate-screw osteosynthesis via an anterolateral approach. There was rapid improvement in vision after surgery, and no complication was observed.

Our case and previous case reports reveal that FES may present a wide range of symptoms and signs that may not fit the classical diagnostic criteria and Bergman's triad of respiratory distress, neurological impairment, and skin rash.
In conclusion, classical diagnostic findings may not be present in all patients with cerebral fat embolism. Cortical blindness may be the novel symptom of CFES. Neurological dysfunction may be initial symptoms of systemic fat embolism and followed by other findings. Cranial DWI and MRI findings are typical and efficiently support the diagnosis. A rapid work-up and exclusion of other possible causes of cerebral microembolism supply a proper diagnose of CFES. Early diagnosis and the treatment of the disease is important for disabling and fatal complications of FES. A favorable outcome is expected after proper supportive medications and rapid fixation of the fracture.

\section{Declaration of conflicting interests}

The authors declared no conflicts of interest with respect to the authorship and/or publication of this article.

\section{Funding}

The authors received no financial support for the research and/or authorship of this article.

\section{REFERENCES}

1. Morales-Vidal SG. Neurologic complications of fat embolism syndrome. Curr Neurol Neurosci Rep 2019;19:14.

2. Rodríguez-Gutiérrez R, Rodarte-Shade M, GonzálezGonzález JG, Lavalle-González FJ. Bergman's triad: Fat embolism syndrome. Am J Med Sci 2015;349:186.

3. Godoy DA, Di Napoli M, Rabinstein AA. Cerebral fat embolism: Recognition, complications, and prognosis. Neurocrit Care 2018;29:358-65.

4. Timon C, Keady C, Murphy CG. Fat embolism syndrome - A qualitative review of its incidence, presentation, pathogenesis and management. Malays Orthop J 2021;15:1-11.

5. Milroy CM, Parai JL. Fat embolism, fat embolism syndrome and the autopsy. Acad Forensic Pathol 2019;9:136-54.

6. Gurd AR, Wilson RI. The fat embolism syndrome. J Bone Joint Surg [Br] 1974;56:408-16.

7. Lim SF, Chong CK, Ng P, Koh S. Transient post-traumatic cortical blindness due to bilateral occipital lobe infarcts in a multiply-injured patient: A case report. Injury Extra 2013;44:54-7.

8. Meena UK, Lamoria RK, Millan RK, Agarwal P, Singh M, Bansal MC. Cortical blindness along with motor aphasia: An unusual presentation of fat embolism syndrome. J Clin Orthop Trauma 2016;7(Suppl 1):17-21.

9. Mellor A, Soni N. Fat embolism. Anaesthesia 2001;56:14554.

10. Schemitsch EH, Jain R, Turchin DC, Mullen JB, Byrick RJ, Anderson GI, et al. Pulmonary effects of fixation of a fracture with a plate compared with intramedullary nailing. A canine model of fat embolism and fracture fixation. J Bone Joint Surg [Am] 1997;79:984-96. 